\title{
Imaging Based Methods of Liver Fibrosis Assessment in Viral Hepatitis: A Practical Approach
}

\author{
Hicham Khallafi ${ }^{1}$ and Kamran Qureshi ${ }^{2}$ \\ ${ }^{1}$ Division of Gastroenterology, Case Western Reserve University, School of Medicine, MetroHealth System, \\ 2500 MetroHealth Drive, Cleveland, OH 44109, USA \\ ${ }^{2}$ Section of Gastroenterology and Hepatology, Temple University Lewis Katz School of Medicine, \\ Temple University Health System, 3440 N. Broad Street, Philadelphia, PA 19140, USA \\ Correspondence should be addressed to Hicham Khallafi; hkhallafi@metrohealth.org
}

Received 20 September 2015; Revised 10 November 2015; Accepted 11 November 2015

Academic Editor: Massimiliano Lanzafame

Copyright ( $\odot 2015$ H. Khallafi and K. Qureshi. This is an open access article distributed under the Creative Commons Attribution License, which permits unrestricted use, distribution, and reproduction in any medium, provided the original work is properly cited.

\begin{abstract}
Liver fibrosis represents the repair mechanism in liver injury and is a feature of most chronic liver diseases. The degree of liver fibrosis in chronic viral hepatitis infections has major clinical implications and presence of advanced fibrosis or cirrhosis determines prognosis. Treatment initiation for viral hepatitis is indicated in most cases of advanced liver fibrosis and diagnosis of cirrhosis entails hepatology evaluation for specialized clinical care. Liver biopsy is an invasive technique and has been the standard of care of fibrosis assessment for years; however, it has several limitations and procedure related complications. Recently, several methods of noninvasive assessment of liver fibrosis have been developed which require either serologic testing or imaging of liver. Imaging based noninvasive techniques are reviewed here and their clinical use is described. Some of the imaging based tests are becoming widely available, and collectively they are shown to be superior to liver biopsy in important aspects. Clinical utilization of these methods requires understanding of performance and quality related parameters which can affect the results and provide wrong assessment of the extent of liver fibrosis. Familiarity with the strengths and weaknesses of each modality is needed to correctly interpret the results in appropriate clinical context.
\end{abstract}

\section{Introduction}

Liver fibrosis is a common pathway of liver injury for multiple chronic liver conditions. Chronic viral hepatitis, metabolic, autoimmune, and cholestatic liver diseases amongst others can lead to clinically significant fibrosis. Assessment of the severity of hepatic fibrosis is essential for determining the prognosis of patients with chronic viral hepatitis and estimating the urgency of antiviral therapy [1]. The severity of liver fibrosis and advanced liver disease dictates the need for further evaluations, such as hepatocellular carcinoma surveillance and esophageal varices screening. Although in several cases the diagnosis of advanced liver fibrosis or liver cirrhosis can be made clinically, the liver biopsy has been used as the conventional reference method for staging fibrosis and diagnosis of liver cirrhosis.

Liver biopsy offers histologic visualization of the extent of liver fibrosis and thus is considered the "best" standard for fibrosis staging and is universally accepted; however it is an invasive technique with associated morbidity. Minor complications are relatively frequent and about $25 \%$ of patients undergoing liver biopsy have pain in the right upper quadrant or right shoulder after the procedure [2]. Severe complications are uncommon; significant bleeding incidents range from $0.05 \%$ to $5.3 \%$ and mortality of less than $0.15 \%$ was seen in the largest series [3]. The accurate evaluation of fibrosis using liver biopsy is also complicated by sampling error and interobserver variation in staging, particularly when inadequate sampling occurs [4]. A study which included 124 patients with chronic Hepatitis C Virus (HCV) infection who underwent simultaneous laparoscopy guided biopsies of the right and left hepatic lobes showed that $33.1 \%$ of subjects were graded to have a difference of at least one stage between the two lobes [5]. Because liver biopsy has several limitations, namely, sampling error and 


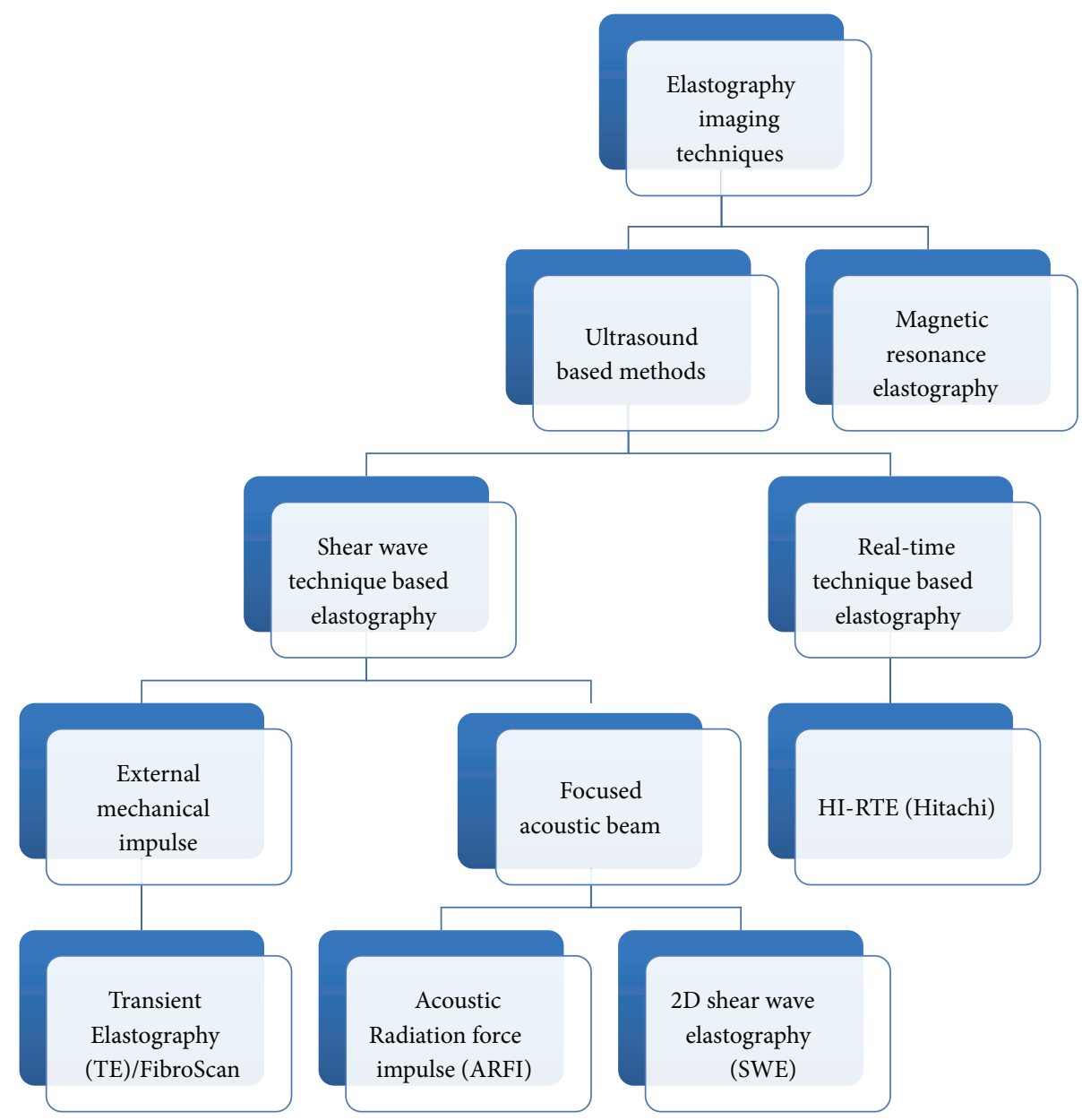

FIGURE 1: Imaging based elastography techniques for liver fibrosis assessment.

intra- and interobserver variability, the Area Under Receiving Operating Characteristic (AUROC) curve values of $>0.9$ for liver biopsy could not be attained in clinical studies for fibrosis staging [6], which is a characteristic for an ideal diagnostic test [7]. Rather than the conventional label of being the "gold" standard, liver biopsy is increasingly recognized as the "best" standard for liver fibrosis staging. In addition, performing liver biopsies on a large scale for clinical use may not be a reasonable, cost-effective, or practical approach. Currently, some of the noninvasive methods of liver fibrosis assessment have better diagnostic value than liver biopsy in chronic liver diseases and are safe to perform. As a result, the noninvasive methods are increasingly replacing invasive liver biopsy in clinical practice due to patient wariness of the morbidity associated liver biopsy and physician ease of clinical use with accuracy.

The noninvasive methods of liver fibrosis can be broadly divided into two main groups: serum biomarkers (which require blood sample collection and estimation and calculation of score) and the imaging based methods. In this review, we will focus on imaging based methods and describe the technical aspects, performance accuracy, meaningful utilization, and incorporation in clinical practice of now available elasticity based methods of liver fibrosis assessment (elastography).

\section{Elastography Imaging}

The techniques included can be divided into four main groups: (A) shear wave elastography technique based on mechanically generated impulse that includes Transient Elastography (FibroScan); (B) shear wave elastography techniques based on the acoustic beam that includes $2 \mathrm{D}$ shear wave elastography/point shear wave elastography/acoustic radiation force impulse; (C) real-time technique based elastography; and (D) magnetic resonance elastography. These are shown in Figure 1 and overview of comparison of various methods is described in Table 1. It is now recommended that every patient with viral hepatitis should have liver disease staging at least once by noninvasive methods [8]. Transient Elastography (FibroScan) is the most extensively used and validated method for fibrosis staging. Shear wave elastography/acoustic radiation force impulse seems promising; however its incorporation in clinical decision seems to be in early stage with only relatively recent pilot studies. Magnetic resonance elastography is not widely used given the 
TABLE 1: Salient features of technical aspects of liver elastography modalities.

\begin{tabular}{|c|c|c|c|c|c|}
\hline Technique & $\mathrm{TE}$ & ARFI & SWE & RTE & MRE \\
\hline $\begin{array}{l}\text { Liver morphologic } \\
\text { examination/image }\end{array}$ & $\begin{array}{l}\text { Unable because of } \\
\text { lack of visualization }\end{array}$ & $\begin{array}{c}\text { Available as } \\
\text { integrated with } \\
\text { ultrasound } \\
\end{array}$ & $\begin{array}{c}\text { Available as } \\
\text { integrated with } \\
\text { ultrasound } \\
\end{array}$ & $\begin{array}{c}\text { Available as } \\
\text { integrated with } \\
\text { ultrasound }\end{array}$ & $\begin{array}{c}\text { Best as } \\
\text { integrated with } \\
\text { MRI }\end{array}$ \\
\hline Type of force & Dynamic & Dynamic & Dynamic & Static & Dynamic \\
\hline Applied Force & $\begin{array}{l}\text { External mechanical } \\
\text { impulse }\end{array}$ & $\begin{array}{c}\text { Internal acoustic } \\
\text { beam }\end{array}$ & $\begin{array}{l}\text { Multiple internal } \\
\text { acoustic beams }\end{array}$ & Heart beat & $\begin{array}{c}\text { External driver } \\
\text { device }\end{array}$ \\
\hline Region of Interest (ROI) & $\begin{array}{l}\text { Monodimensional } \\
\text { ROI: } 1 \times 4 \mathrm{~cm} \text { area }\end{array}$ & $\begin{array}{l}\text { Monodimensional } \\
\text { ROI: } 1 \times 0.5 \mathrm{~cm}\end{array}$ & $\begin{array}{l}\text { Multiple real-time } \\
\text { color-coded elasticity } \\
\text { imaging: } 2 \mathrm{D} \text { images } \\
\text { ROI: up to } 5 \mathrm{~cm} \times 5 \mathrm{~cm}\end{array}$ & $\begin{array}{c}\text { Not a } \\
\text { quantitative } \\
\text { approach: } \\
\text { "Relative } \\
\text { Elasticity" }\end{array}$ & $\begin{array}{c}\text { Evaluates the entire } \\
\text { liver with } 2 \mathrm{D}-3 \mathrm{D} \\
\text { images }\end{array}$ \\
\hline Measurement results (LSM & $\begin{array}{l}\text { Quantitative results } \\
\text { displayed in } \mathrm{kPa}\end{array}$ & $\begin{array}{l}\text { Quantitative results } \\
\text { displayed often in } \mathrm{m} / \mathrm{s}\end{array}$ & $\begin{array}{c}\text { Quantitative results } \\
\text { displayed in } \mathrm{kPa} \text { or } \mathrm{m} / \mathrm{s} \\
\text { at a wide range of values }\end{array}$ & Qualitative & $\begin{array}{c}\text { Quantitative } \\
\text { results displayed in } \\
\mathrm{kPa}\end{array}$ \\
\hline
\end{tabular}

described limitations despite the higher performance. Major advantage of these imaging methods is that these can be conveniently repeated over time in patients who are treated or those who stay on long term treatment for viral hepatitis. The LSM can be followed to monitor for therapy response and improvement of liver fibrosis. In addition, one can expect that the elastography improvement over time will predict improvement in clinical outcomes.

\subsection{Transient Elastography (TE). TE or Vibration Controlled} Transient Elastography technique is exclusively used in FibroScan which was developed nearly 10 years ago by Echosens [9]. It is now the reference test and is the best validated technique for noninvasive evaluation of liver fibrosis. Its use in the United States was approved by the Food and Drug Administration (FDA) in 2013 for liver fibrosis assessment in HCV patients.

2.1.1. Technical Aspects. TE uses mechanically generated elastic shear wave (SW) and follows its propagation through the liver to measure its speed in a single dimension. The speed of SW in the liver is directly related to its stiffness and the level of fibrosis (elastic modulus) [9]. A probe transducer is used which mechanically generates SW by vibration at the frequency of $50 \mathrm{~Hz}$ and is placed on the chest wall in the right ninth to eleventh intercostal space. $\mathrm{SW}$ is followed in the cylindrical Region of Interest (ROI) of $1 \mathrm{~cm} \times 4 \mathrm{~cm}$ dimension, 2.5 or $6.5 \mathrm{~cm}$ below the skin surface to estimate the speed by use of a sensor. A reading is obtained each time button is pressed while probe is in a correct position and is called liver stiffness measurement (LSM), expressed in kilopascals $(\mathrm{kPa})$. The LSMs range from 2.5 to $75 \mathrm{kPa}$, with mean value around $5 \mathrm{kPa}$ [9]. Accurate result of an examination requires careful interpretation of data from at least 10 valid LSMs. A success rate (the ratio of valid measurements to the total number of measurements) of $>60 \%$ and an interquartile range (IQR), which reflects variations among measurements, of $<30 \%$ of the median LSM value (i.e., IQR/median $\mathrm{LSM} \leq 30 \%$ ) are considered as an adequate study [10]. Table 1 compares certain technical aspects in comparison to other elastography techniques.

2.1.2. Diagnostic Performance and Accuracy. Since the introduction of TE in 2005 in France, its use in early European cohorts of HCV patients has clearly shown that TE can detect clinically significant fibrosis and cirrhosis with a good accuracy and reproducibility $[11,12]$. There have been several validation studies in $\mathrm{HCV}$ patients indicating that LSM strongly correlates with liver fibrosis on biopsy. Furthermore, in the largest multicenter independent study to date, TE outperformed all other noninvasive tests for the diagnosis of cirrhosis [13]. Similar findings were noted in multiple North American publications. In a recent large United States multicenter cohort study, TE was evaluated in patients with chronic type B and $C$ viral hepatitis (903 patients), and the readings correlated strongly with the fibrosis stage on biopsy with AUROC of 0.89 and 0.92 for significant fibrosis ( $\mathrm{F} \geq$ 2) and F4, respectively, with good intra- and interoperator reproducibility (Interclass Correlation (ICC) of 0.95 and $0.99)$. Liver stiffness cutoff values in this study $(\mathrm{kPa})$ were 8.4 for $\geq \mathrm{F} 2$ and 12.8 for F4 [14]. TE generated LSMs have been increasingly applied to other chronic liver diseases besides HCV that include Chronic Hepatitis B Virus (HBV) infection, Nonalcoholic Steatohepatitis (NASH), Alcoholic Liver Disease (ALD), and cholestatic liver diseases [15, 16]. Different liver disease specific stiffness $\mathrm{KPa}$ cutoffs were described and are presented in Table 2 . Similar to previously noted multiple systemic reviews and meta-analyses, recent large systematic review of published studies between 2001 and June 2011 across chronic liver diseases showed a strong diagnostic accuracy of TE compared to biopsy stages with an AUROC of $0.88,0.92$, and 0.94 for $F \geq 2, F \geq 3$, and F4, respectively [17].

2.1.3. Prognosis and Longitudinal Follow-Up. There is good evidence to support the association between TE measurements and survival. A French study examining 5-year survival in a large cohort of $1457 \mathrm{HCV}$ patients demonstrated that the overall survival using TE for LSM $\leq 9.5 \mathrm{kPa}$ was $96 \%$; 
TABle 2: Diagnostic use of Transient Elastography in literature.

\begin{tabular}{|c|c|c|c|c|c|c|}
\hline & \multirow{2}{*}{ Patients $(n)$} & \multirow{2}{*}{ Diseases } & \multicolumn{2}{|c|}{ AUROC } & \multicolumn{2}{|c|}{ Cutoff $\mathrm{kPa}$} \\
\hline & & & $\geq \mathrm{F} 2$ & $=\mathrm{F} 4$ & $\geq \mathrm{F} 2$ & $\mathrm{~F} 4$ \\
\hline Ziol et al. [12] & 251 & $\mathrm{HCV}$ & 0.79 & 0.97 & 8.8 & 14.6 \\
\hline Castéra et al. [10] & 183 & $\mathrm{HCV}$ & 0.83 & 0.95 & 7.1 & 12.5 \\
\hline Marcellin et al. [15] & 173 & $\mathrm{HBV}$ & 0.81 & 0.93 & 7.2 & 11.0 \\
\hline Wong et al. [16] & 246 & $\mathrm{NASH}$ & 0.80 & 0.94 & 7 & 10.3 \\
\hline Fraquelli et al. [50] & 200 & All liver diseases & 0.84 & 0.90 & 7.9 & 11.9 \\
\hline Degos et al. [13] & 1839 Multicenter & All liver diseases & 0.76 & 0.90 & 5.6 & 12.9 \\
\hline Afdhal et al. [14] & 907 Multicenter & $\mathrm{HCV} / \mathrm{HBV}$ & 0.89 & 0.92 & 8.4 & 12.8 \\
\hline Steadman et al. [17] & Meta-analysis & All liver diseases & 0.88 & 0.94 & 一 & - \\
\hline
\end{tabular}

for $>9.5 \mathrm{kPa}$ was $77 \%$; for $>20 \mathrm{kPa}$ was $66 \%$; for $>30 \mathrm{kPa}$ was $57 \%$; for $>40 \mathrm{kPa}$ was $47 \%$; and for $>50 \mathrm{kPa}$ was $42 \%$. TE had superior diagnostic performance for predicting 5-year survival compared with biopsy [18]. Similar findings were noted in a United States based study with 667 patients with various underlying liver conditions, in which Klibansky et al. reported excellent diagnostic performance of TE for predicting a composite outcome including death, decompensation, and HCC (AUROC of 0.87) [19]. A recent large cohort study (1,025 patients were included) provided strong evidence of the clinical utility of serial TE examinations [20]. Excellent survival from a liver related mortality/transplantation rate of $\leq 1.2 \%$ over 3 years was observed in three groups of patients: (group 1) $\mathrm{LSM} \leq 7 \mathrm{kPa}$ regardless of response to antiviral therapy; (group 2) LSM $\geq 7 \mathrm{kPa}$ with sustained virological response; (group 3) LSM 7-14 kPa with a change of LSM $\leq$ $1 \mathrm{kPa} /$ year. As compared to liver related mortality of $6.6-$ $10.4 \%$ in patients with a baseline LSM $\geq 14 \mathrm{kPa}$ or LSM 7$14 \mathrm{kPa}$ with a change of $\mathrm{LSM} \geq 1 \mathrm{kPa} /$ year, the worse prognosis $(21.4 \%)$ was in the group with a baseline LSM $\geq 14 \mathrm{kPa}$ and any increase of LSM over 3 years [20].

2.1.4. Technical Limitations and Training. In the large Castera series (13,369 VCTE examinations reported), the TE examination failure rate and unreliable LSM readings occurred in $3.1 \%$ and $15.8 \%$, respectively, and they were mostly associated with BMI greater than $30 \mathrm{~kg} / \mathrm{m}^{2}$ and operator inexperience [21].

$B M I$. To reduce the number of patients with unreliable readings related to $\mathrm{BMI}$, several probe types have been developed. The XL probe is useful in subjects in whom a valid LSM cannot be obtained with the $M$ probe. Myers et al. reported that the failure rate decreased from $59 \%$ for the $\mathrm{M}$ probe to $4.9 \%$ by using the XL probe for patients with BMI over $40 \mathrm{~kg} / \mathrm{m}^{2}$ [22]. LSMs taken with the XL probe were noted to be lower than those obtained by the $\mathrm{M}$ probe by a median of $1.4 \mathrm{kPa}$ [23].

Operator Experience. Operator inexperience, defined as $<500$ examinations, was initially seen to be independently associated with both LSM failure and reading unreliability. Degos et al. demonstrated that there was no difference in the performance of TE between physician performed TE and a trained technician as long as adequate experience was acquired [13]. Kettaneh et al. demonstrated that a reasonable performance for the diagnosis of cirrhosis could be achieved with as few as 50 examinations; the authors conclude the FibroScan may be used in nonspecialized units [24]. United Stated FDA and FibroScan manufacturer agreement requires that an operator undergo a training course and perform a minimum of 10 cases under supervision of a proctor before getting certified to use the device independently.

Other Factors. Liver stiffness is not synonymous with fibrosis and any process that may change hepatic viscoelastic properties can affect LSM readings and lead to overestimation of liver stage. The most important LSM modifying factors include acute inflammation (elevated ALT) [25], extrahepatic cholestasis [26], passive cardiogenic congestion [27], and food intake [28]. Arena et al. showed that after a meal the liver stiffness returned to baseline levels within $120 \mathrm{~min}$ in all patients independent of fibrosis stage [28]. Patients without significant fibrosis but high levels of ALT could get LSM within the range for cirrhosis. This was also noted in a large Canadian multicenter study in which the authors reported that, for every $100 \mathrm{IU} / \mathrm{mL}$ increase in ALT, LSM increased by $1.1 \mathrm{kPa}[29]$.

2.1.5. Access and Reimbursement in the United States. Since January 2015, a new code 91200 was added for liver elastography performed via mechanically induced SW technique, such as vibration. The code includes coverage for examination and interpretation. Current status shows that only the TE/FibroScan is billable and has been reimbursed. Some insurers may require prior authorization. Medicare reimbursement was reported to approach $\$ 134.8$, while the additional facility fees were not included.

2.1.6. Clinical Utilization in Viral Hepatitis. TE is well validated in viral hepatitis and its diagnostic performance was found to be equally powerful in HBV, HCV, and HIV-HCV coinfection. However, it is important to use the LSM obtained via TE in appropriate clinical context. The following provides a useful checklist prior to interpretation and use of TE readings. 


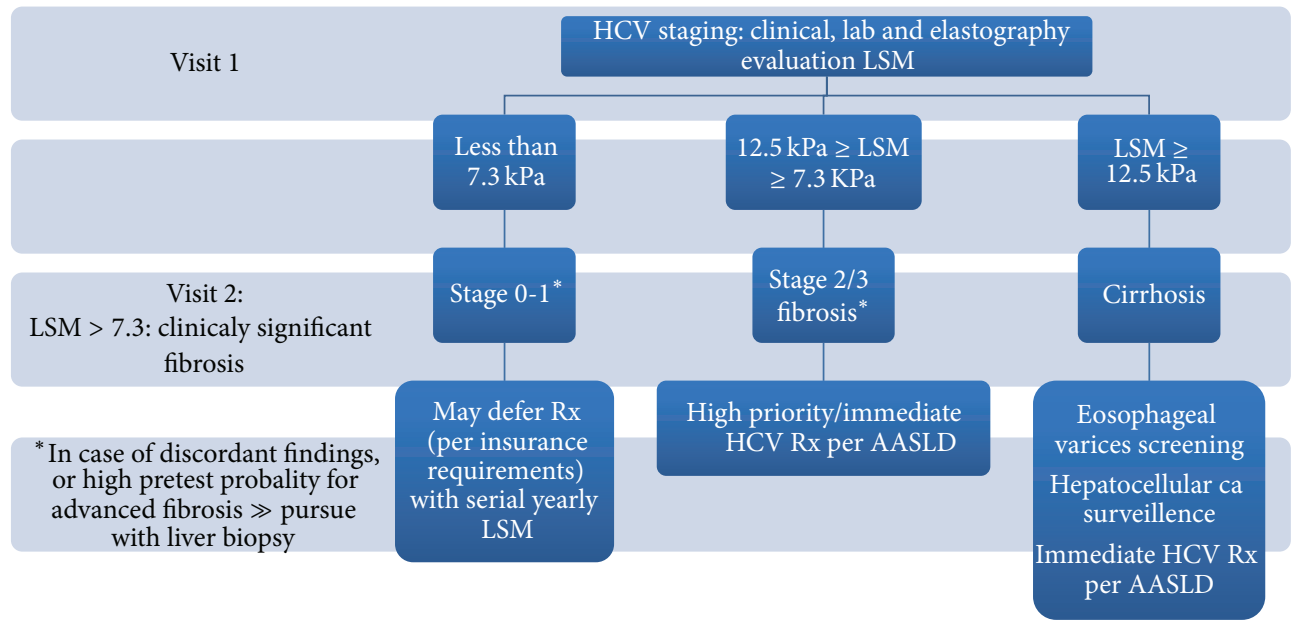

FIGURE 2: Algorithm for the use of TE for liver staging in HCV and HIV-HCV coinfection.

Transient Elastography Clinical Use Checklist for Reliable Interpretation of Results

(i) Operator experience:

(a) Ideally more than 100 examinations.

(b) Standardized protocol of examination.

(ii) Patient characteristics:

(a) Patient diagnosis: HCV, HBV, NASH, or other liver diseases (for use of different cutoffs for diagnosis of cirrhosis).

(b) Obesity: use of XL probe (2.5 MHz transducer) for $\mathrm{BMI}>30 \mathrm{~kg} / \mathrm{m}^{2}$ or for thick chest wall (skin to liver surface distance $>2.5 \mathrm{~cm}$ ).

(c) Absence of ascites: on clinical or radiologic examination.

(d) Fasting status for at least 2 hours.

(e) Absence of active alcohol use.

(f) Absence of right heart failure/hepatic congestion.

(iii) Biochemical parameters:

(a) ALT levels: should be less than 5 times ULN for reliable readings, also for use of ALT levels based cutoffs (for HBV).

(b) Hepatitis B serology: e antigen status (HBV).

(c) Serologic noninvasive fibrosis testing: for intermediate results (HCV).

(iv) Reliable readings:

(a) Valid readings (shots): at least 10 .

(b) Success rate (valid shots/total number of shots) of $>60 \%$.

(c) Variability in valid shots reading (IQR/median LSM) of less than $30 \%$.
It is important to recognize that the clinical confounding variables in clinical decision-making are important (obesity, inflammation, and cholestasis). This entails that a physical examination is done including BMI estimation and assessment of presence of ascites; liver functional and biochemical testing (ALT, alkaline phosphatase) should be available at the time of LSM evaluation. Patient should be fasting for at least 2 hours prior to performing the test. LSMs are a section of the overall diagnosis process and must take into account all the disease clinical, laboratory, and radiological findings. Appreciating the TE intrinsic validities (positive and negative predictive value and AUROCs) and the pretest probability of advanced fibrosis would facilitate the decision-making process.

$H C V$ including $H I V-H C V$ Coinfection. TE can identify advanced fibrosis with an excellent accuracy with AUC $>0.9$, which was replicated across multiple cohorts, and particularly for the diagnosis of cirrhosis. TE mainly categorizes HCV patients in 2 subsets distinguishing patients with cirrhosis from patients with early fibrosis. Patients in the intermediate fibrosis range may need liver biopsy if accurate staging allocation is needed. Multiple clinical decision algorithms have been developed and can significantly reduce the need for liver biopsy. Boursier et al. showed that biomarkers noninvasive tools in conjunction with FibroScan liver stiffness evaluation could obviate the need for liver biopsy with over $86.7 \%$ accuracy [30]. One suggested algorithm for staging of liver disease in HCV is shown in Figure 2. The current recommendations for treatment of HCV differ considerably between healthcare systems and insurance policies. TE can be used to prioritize patients for HCV therapy based on liver disease stage. TE should be combined with serologic noninvasive fibrosis assessment tests to increase the diagnostic accuracy for significant fibrosis and thus initiation of therapy. Once access to care for $\mathrm{HCV}$ widens to nonliver or infectious disease providers TE can be used to estimate the presence of cirrhosis and thus referral for specialized care can be suggested [31]. Studies have confirmed histologic improvement and thus 
clinical outcomes in patients who are cured of HCV infection after therapy $[32,33]$. Liver elasticity improves with HCV cure and can be measured as LSM by TE. Several studies showed improvement in LSM after HCV treatment [34, 35]. However, care should be taken while interpreting the follow-up results of TE, as improvement in ALT with HCV treatment will have an impact on LSM. The clinical utility of improvement in LSM after SVR has a little clinical significance in patients without cirrhosis; however the determination of cirrhosis regression after SVR in HCV treated patients could be of important clinical implication. The study that evaluated the cirrhosis estimation showed sensitivity of $61 \%$ when the usual cutoff of $12 \mathrm{kPa}$ for cirrhosis was used [36] in patients with cirrhosis who achieved SVR after treatment. This indicates that TE may not be a good tool to estimate cirrhosis regression in HCV patients after SVR, and the time interval between LSM assessments after obtaining SVR is not yet tested. TE showed good predictability, however, in regard to liver related outcomes in HCV patients. In patients who were untreated, a large study showed that gradually rising LSM by $1 \mathrm{kPa}$ per year in HCV patients portends poor clinical outcomes [20]. Other studies were also able to stratify HCV patients based on LSM cutoff values as those with increased risk of long term liver related outcomes $[18,19]$.

$H B V$. Overall TE performs better than the serologic noninvasive methods for diagnosing advanced liver fibrosis in HBV infected patients [37]. Chronic HBV infection has various phases of liver injury, inflammation, and viral replication during a lifetime and TE can be used effectively to estimate the extent of liver fibrosis. TE can yield falsely high readings in patients with elevated liver enzymes (ALT levels), either during active hepatitis phase or during reactivation flares and even 3-6 months after ALT normalization following an acute exacerbation of chronic HBV infection [38]. For HBeAg negative patients who are in immune inactive phase (inactive carriers) TE showed steady results [39] as compared with other methods and LS $<5-6 \mathrm{kPa}$ suggested absence of or minimal fibrosis [40]. LSM readings higher than $12 \mathrm{kPa}$ have good predictability to diagnose cirrhosis in $\mathrm{HBV}$ inactive carriers [41], while intermediate LSM values should be followed up with a liver biopsy for correct estimation of liver fibrosis [42]. For $\mathrm{HBeAg}$ negative patients in immune active phase and fluctuating or persistent borderline HBV viremia TE may be preferred over performing a liver biopsy to diagnose cirrhosis [43] or advanced fibrosis [42], respectively. Among HBeAg positive patients with normal or mildly elevated ALT, using TE can be helpful in estimation of liver fibrosis in adults to distinguish between immune tolerant phase and advance liver disease secondary to undiagnosed immune active phase of liver injury [44]. The estimation of liver fibrosis by TE can avoid liver biopsy in many patients and guide initiation of antiviral therapy in a timely manner in chronic $\mathrm{HBV}$ infection. TE should be considered to diagnose cirrhosis in patient and hence to start therapy with chronic HBV infection and clinical suspicion of advanced liver disease despite normal ALT. Long term antiviral therapy has shown resolution of liver fibrosis/regression of cirrhosis in $\mathrm{HBV}$ infected patients [45]. Several studies have shown that TE can be used for follow-up and documentation improvement in liver fibrosis while on HBV therapy $[46,47]$. The follow-up LSM readings should be compared with the baseline reading taken once ALT normalize after introduction of antivirals to avoid confounding effect of raised ALT on TE readings [48]. The prognostic utility of LSM obtained by TE in HBV patients in regard to liver related outcomes and survival is being evaluated. One study showed increased risk of HCC in HBV patients who were determined to have cirrhosis by TE assessment [49].

2.2. 2D Shear Wave Elastography (SWE). Cleared by the FDA in December 2014, SWE technique is based on the combination of SW induced by multiple focused acoustic beams and a very rapid acquisition of ultrasound images (up to 20,000 images per second). In contrast to TE, an acoustic SW is generated and evaluated in SWE which can be coupled to the standard liver ultrasound examination for stiffness measurements. Multiple ultrasound systems that have SWE capabilities are commercially available in the United States, including those by Supersonic Imagine, SA (Aix-enProvence, France; Ultrafast), and Logiq E9 ultrasound system (GE Healthcare, WI, USA).

2.2.1. Technical Aspects. This application is able to capture the propagation of the resulting SW in real time and in a large area of liver parenchyma, developing real-time colorcoded elasticity imaging. Quantitative measurements can be performed in the color window by placing one or more ROI inside the sample box. The ROI of SWE is fan-shaped and larger than other modalities (up to $50 \mathrm{~mm} \times 50 \mathrm{~mm}$ ). Results are given in $\mathrm{m} / \mathrm{s}$ or $\mathrm{kPa}$ [58]. During the B-mode sonographic examination of the liver, a standardized ROI box is positioned in a predetermined anatomical site within the liver parenchyma for evaluation. The SW is generated from a focused ultrasound beam in the vicinity of the designated ROI. The velocity of the wave propagation, expressed in meters per second $(\mathrm{m} / \mathrm{s})$ or kilopascals $(\mathrm{KPa})$, is calculated and allows LSM. The measurements are not limited by the presence of ascites as the ultrasound beam generating the SW propagates through fluids. The stiffer the liver is, the higher the recorded SW velocity is [59].

2.2.2. Diagnostic Performance and Accuracy. As the application of SWE to liver fibrosis is a relatively newer approach, there are only a few studies evaluating its accuracy in liver diseases (Table 3). Poynard et al. in the largest liver SWE study to date (422 patients) showed the performance of SWE for the diagnosis of cirrhosis was similar to those of TE except in patients with ascites [52]. The better performance of SWE in patients with ascites may not be important clinically. Another recent single center prospective study with 349 consecutive patients with chronic liver diseases compared the liver stiffness measurements obtained by SWE and TE with liver biopsy [53]. In this study AUROCs of SWE, TE were, respectively, $0.89,0.86$ for the diagnosis of mild fibrosis; $0.88,0.84$ for the diagnosis of significant fibrosis; $0.93,0.87$ for the diagnosis of severe fibrosis; $0.93,0.90$ for the 
TABLE 3: Diagnostic use of acoustic impulse based techniques of elastography in literature.

\begin{tabular}{|c|c|c|c|c|c|c|}
\hline & \multirow{2}{*}{ Patients $(n)$} & \multirow{2}{*}{ Liver disease } & \multicolumn{2}{|c|}{ AUROC } & \multicolumn{2}{|c|}{ Cutoff $\mathrm{KPa}(\mathrm{SWE})$ and $\mathrm{m} / \mathrm{s}$ (ARFI) } \\
\hline & & & $\geq \mathrm{F} 2$ & $=\mathrm{F} 4$ & $\geq \mathrm{F} 2$ & $=\mathrm{F} 4$ \\
\hline Ferraioli et al. (SWE) [51] & $\begin{array}{c}138 \\
\text { Single center }\end{array}$ & $\mathrm{HCV}$ & 0.92 & 0.98 & 7.1 & 10.4 \\
\hline Poynard et al. (SWE) [52] & $\begin{array}{c}422 \\
\text { Single center }\end{array}$ & $\mathrm{HCV}$ & 0.66 & 0.74 & 8.8 & 14.5 \\
\hline Cassinotto et al. (SWE) [53] & $\begin{array}{c}349 \\
\text { Single center }\end{array}$ & All liver diseases & 0.88 & 0.93 & 8 & 10.7 \\
\hline Friedrich-Rust et al. (ARFI) [54] & 518 & All liver diseases & 0.87 & 0.93 & 1.35 & 1.8 \\
\hline Sporea et al. (ARFI) [55] & 914 & $\mathrm{HCV}$ & 0.79 & 0.84 & 1.33 & 1.55 \\
\hline Bota et al. (ARFI) [56] & 1163 & All liver diseases & 0.85 & 0.93 & 1.31 & 1.80 \\
\hline Nierhoff et al. (ARFI) [57] & 3,951 & All liver diseases & 0.84 & 0.91 & 1.37 & 1.87 \\
\hline
\end{tabular}

diagnosis of cirrhosis. SWE had a higher accuracy than TE for the diagnosis of severe fibrosis $(\geqslant F$ 3) but no statistically significant difference was observed for the diagnosis of mild fibrosis and cirrhosis [53]. The initial United States study was a pilot program that was recently published in September 2014. In this prospective study of 50 patients with chronic liver disease, the SWE measurements were compared to liver biopsy with a good accuracy for advanced fibrosis [60]. SWE is a new approach that has been applied to liver stiffness measurement. However its accuracy and performance in liver diseases need further validation in larger multicenter studies.

2.2.3. Technical Limitations. With SWE only a few diseases specific LSMs are reported. The bulk of the studies have been performed in patients with HCV; therefore these cutoffs may not be applicable to other liver conditions. Only small series of patients with NAFLD have been reported and few studies addressing the confounding factors have been published. Another challenge in interpreting and obtaining data is a lack of standardization.

2.3. Acoustic Radiation Force Impulse (ARFI). Cleared by the FDA in 2013, ARFI is a quantitative technique that provides a single dimensional LSM of predetermined sample box in liver. Similar to SWE, ARFI can be performed at the time of the standard ultrasound evaluation (Table 3 ). The ROI is smaller than SWE: $1 \times 0.5 \mathrm{~cm}$ rectangle. The LSM is expressed in $\mathrm{m} / \mathrm{s}$, which is reflective of the SW velocity travelling perpendicular to the source [61]. ARFI is developed by Philips Healthcare (Bothell, WA, USA; ElastPQ) and Siemens Medical Solutions (Mountain View, CA, USA; Virtual Touch Tissue Quantification (VTTQ)).

2.3.1. Diagnostic Performance and Accuracy. ARFI was initially validated in a large international multicenter study (10 centers, 5 countries) of $914 \mathrm{HCV}$ patients [55]. The ARFI was compared to liver biopsy and TE; the LSM obtained by the ARFI correlated with Metavir stage with AUROC of 0.792 and 0.842 for significant fibrosis $(\mathrm{F} \geq 2)$ and $F 4$, respectively [55]. LSM cutoff values in this study were $1.33 \mathrm{~m} / \mathrm{s}$ for $\geq \mathrm{F} 2$ and $1.55 \mathrm{~m} / \mathrm{s}$ for F4. The correlation with histological fibrosis was not significantly different for TE in comparison with ARFI; however TE was better for predicting the presence of liver cirrhosis $(P=0.01)$ than ARFI. A meta-analysis which included 13 studies with 1163 patients and various chronic liver diseases compared ARFI to liver biopsy and TE [56]. The authors showed that ARFI is a good method for assessing liver fibrosis, with AUROC of 0.85 for detecting significant fibrosis and 0.93 for diagnosing cirrhosis with a predictive value similar to TE. Similarly, another meta-analysis, the largest to date (36 studies with 3,951 patients), showed the diagnostic accuracy expressed as AUROC of 0.84 and 0.91 for significant fibrosis and cirrhosis, respectively [57]. Overall, the review of the major published meta-analyses suggests that ARFI is a reliable method for the diagnosis of advanced fibrosis/cirrhosis and likely comparable to TE. However, a wide range of mean values defining cirrhosis was noted contrasting with a narrower range of fibrosis stages cutoffs with FibroScan.

2.4. Real-Time Technique Based Elastography (RTE). This method developed initially by Hitachi Medical Systems, E-Mode, as incorporated into a conventional ultrasound machine is based on strain imaging induced by manual compression. It is a nonquantitative technique which is less accurate in staging liver fibrosis than TE. This technique is not FDA cleared or approved for fibrosis staging.

2.5. Magnetic Resonance Elastography (MRE). MRE was first introduced in the United States by GE Healthcare, based on technology developed at Mayo Clinic (Rochester, MN), and was cleared by the FDA in 2009. This technique is also available on other commercial MRE configurations including Philips Healthcare (2014) and Siemens Medical Solutions (2012).

2.5.1. Technical Aspects. MRE can be coupled to the conventional liver MR imaging protocol and involves 3 steps [62]: (1) propagation of SW within the liver from a driver source generating a continuous $60 \mathrm{~Hz}$ acoustic vibration; (2) imaging the propagating SW in the liver using a MRI 
sequence with motion-encoding gradients; and (3) processing the information in the wave images with an inversion algorithm to generate quantitative elastogram/stiffness maps that measures stiffness in $\mathrm{kPa}$. ROI is placed on the stiffness maps to obtain stiffness values. The ROI permits a larger volume of liver to be sampled. The mean stiffness values reported in the literature range from 2.05 to $2.44 \mathrm{kPa}$, and the range of normal liver stiffness is determined to be between 1.54 and $2.87 \mathrm{kPa}$ [63].

2.5.2. Diagnostic Performance and Accuracy. In a recent published systematic review and meta-analysis (12 studies with 697 patients with chronic liver diseases), the diagnostic performance of MRE was comparable, if not superior, to that of TE and ARFI [64]. The overall diagnostic accuracy of MRE for discriminating advanced fibrosis (> stage 3) was noted to be excellent with an AUROC of 0.93. The mean AUROC values (and 95\% confidence intervals) for the diagnosis of any ( $\geq$ stage 1 ), advanced fibrosis ( $\geq$ stage 3 ), and cirrhosis were as follows: 0.84 (0.76-0.92), 0.93 (0.90-0.95), and 0.92 (0.900.94), respectively [64]. In addition, the authors performed a subgroup analysis; the Body Mass Index (BMI), the degree of liver inflammation, and the underlying etiology of the liver disease did not influence the accuracy of the MRE [64]. Similar results were noted in another meta-analysis in which 11 MRE studies (982 patients) and 15 ARFI studies (2,128 patients) were selected and modalities were compared [65]. Authors concluded that the MRE is more accurate than ARFI particularly in diagnosing early stages of hepatic fibrosis; AUROCs for MRE staging fibrosis were 0.94, 0.97, 0.96, and 0.97 for F1-F4, respectively, whereas AUROCs for ARFI staging were $0.82,0.85,0.94$, and 0.94 for F1-F4, respectively [65]. Excitingly, MRE consistently has been reported as a highly accurate, noninvasive technique for the diagnosis and staging of liver fibrosis and is likely superior to US based techniques across all published studies. This has been noted across most of the liver diseases.

2.5.3. Technical Limitations. As a new technique, many MRE related studies may not have captured all the potential confounding factors. High hepatic iron content may explain the failure rate noted in early MRE evaluations (signal-tonoise limitations). Patient related factors (inadequate breath hold/claustrophobia) and the cost may be additional limitations to the overall acceptance.

\section{Conclusion}

There is an increasing evidence for the diagnostic and prognostic utility of noninvasive methods of estimation of liver fibrosis and cirrhosis in patients with viral hepatitis. It is estimated that the use of noninvasive imaging based methods to assess liver fibrosis will increase tremendously in the near future. Accurate clinical use and understanding of reported findings will help in patient care and reduce the number of and thus morbidity associated with liver biopsies performed. In addition, imaging based methods have greater diagnostic utility than liver biopsy and can effectively be for follow-up assessments and they might have a role in prognostication of clinical liver related complications and mortality.

\section{Conflict of Interests}

The authors declare that there is no conflict of interests regarding the publication of this paper.

\section{References}

[1] American Association for the Study of Liver Diseases, "Recommendations for testing, managing, and treating hepatitis C," 2014, http://www.hcvguidelines.org/full-report-view.

[2] A. I. Tobkes and H. J. Nord, "Liver biopsy: review of methodology and complications," Digestive Diseases, vol. 13, no. 5, pp. 267-274, 1995.

[3] D. C. Rockey, S. H. Caldwell, Z. D. Goodman, R. C. Nelson, and A. D. Smith, "Liver biopsy," Hepatology, vol. 49, no. 3, pp. 10171044, 2009.

[4] I. Siddique, H. Abu El-Naga, J. P. Madda, A. Memon, and F. Hasan, "Sampling variability on percutaneous liver biopsy in patients with chronic hepatitis C virus infection," Scandinavian Journal of Gastroenterology, vol. 38, no. 4, pp. 427-432, 2003.

[5] A. Regev, M. Berho, L. J. Jeffers et al., "Sampling error and intraobserver variation in liver biopsy in patients with chronic HCV infection," American Journal of Gastroenterology, vol. 97, no. 10, pp. 2614-2618, 2002.

[6] S. H. Mehta, B. Lau, N. H. Afdhal, and D. L. Thomas, "Exceeding the limits of liver histology markers," Journal of Hepatology, vol. 50, no. 1, pp. 36-41, 2009.

[7] E. R. DeLong, D. M. DeLong, and D. L. Clarke-Pearson, "Comparing the areas under two or more correlated receiver operating characteristic curves: a nonparametric approach," Biometrics, vol. 44, no. 3, pp. 837-845, 1988.

[8] European Association for Study of Liver and Asociacion Latinoamericana para el Estudio del Higado, "EASL-ALEH Clinical Practice Guidelines: non-invasive tests for evaluation of liver disease severity and prognosis," Journal of Hepatology, vol. 63, no. 1, pp. 237-264, 2015.

[9] L. Sandrin, B. Fourquet, J.-M. Hasquenoph et al., "Transient elastography: a new noninvasive method for assessment of hepatic fibrosis," Ultrasound in Medicine and Biology, vol. 29, no. 12, pp. 1705-1713, 2003.

[10] L. Castéra, J. Vergniol, J. Foucher et al., "Prospective comparison of transient elastography, Fibrotest, APRI, and liver biopsy for the assessment of fibrosis in chronic hepatitis C," Gastroenterology, vol. 128, no. 2, pp. 343-350, 2005.

[11] L. Castera, X. Forns, and A. Alberti, "Non-invasive evaluation of liver fibrosis using transient elastography," Journal of Hepatology, vol. 48, no. 5, pp. 835-847, 2008.

[12] M. Ziol, A. Handra-Luca, A. Kettaneh et al., "Noninvasive assessment of liver fibrosis by measurement of stiffness in patients with chronic hepatitis C," Hepatology, vol. 41, no. 1, pp. 48-54, 2005.

[13] F. Degos, P. Perez, B. Roche et al., "Diagnostic accuracy of FibroScan and comparison to liver fibrosis biomarkers in chronic viral hepatitis: a multicenter prospective study (the FIBROSTIC Study)," Journal of Hepatology, vol. 53, no. 6, pp. 1013-1021, 2010.

[14] N. H. Afdhal, B. R. Bacon, K. Patel et al., "Accuracy of fibroscan, compared with histology, in analysis of liver fibrosis in patients 
with hepatitis B or C: a United States multicenter study," Clinical Gastroenterology and Hepatology, vol. 13, no. 4, pp. 772.e3779.e3, 2015.

[15] P. Marcellin, M. Ziol, P. Bedossa et al., "Non-invasive assessment of liver fibrosis by stiffness measurement in patients with chronic hepatitis B," Liver International, vol. 29, no. 2, pp. 242$247,2009$.

[16] V. W.-S. Wong, J. Vergniol, G. L.-H. Wong et al., "Diagnosis of fibrosis and cirrhosis using liver stiffness measurement in nonalcoholic fatty liver disease," Hepatology, vol. 51, no. 2, pp. 454-462, 2010.

[17] R. Steadman, R. P. Myers, L. Leggett et al., "A health technology assessment of transient elastography in adult liver disease," Canadian Journal of Gastroenterology, vol. 27, no. 3, pp. 149-158, 2013.

[18] J. Vergniol, J. Foucher, E. Terrebonne et al., "Noninvasive tests for fibrosis and liver stiffness predict 5-year outcomes of patients with chronic hepatitis C," Gastroenterology, vol. 140, no. 7, pp. 1970-1979, 2011.

[19] D. A. Klibansky, S. H. Mehta, M. Curry, I. Nasser, T. Challies, and N. H. Afdhal, "Transient elastography for predicting clinical outcomes in patients with chronic liver disease," Journal of Viral Hepatitis, vol. 19, no. 2, pp. e184-e193, 2012.

[20] J. Vergniol, J. Boursier, C. Coutzac et al., "Evolution of noninvasive tests of liver fibrosis is associated with prognosis in patients with chronic hepatitis C," Hepatology, vol. 60, no. 1, pp. 65-76, 2014.

[21] L. Castéra, J. Foucher, P.-H. Bernard et al., "Pitfalls of liver stiffness measurement: a 5-year prospective study of 13,369 examinations," Hepatology, vol. 51, no. 3, pp. 828-835, 2010.

[22] R. P. Myers, G. Pomier-Layrargues, R. Kirsch et al., "Feasibility and diagnostic performance of the FibroScan XL probe for liver stiffness measurement in overweight and obese patients," Hepatology, vol. 55, no. 1, pp. 199-208, 2012.

[23] R. P. Myers, G. Pomier-Layrargues, R. Kirsch et al., "Discordance in fibrosis staging between liver biopsy and transient elastography using the FibroScan XL probe," Journal of Hepatology, vol. 56, no. 3, pp. 564-570, 2012.

[24] A. Kettaneh, P. Marcellin, C. Douvin et al., "Features associated with success rate and performance of fibroscan measurements for the diagnosis of cirrhosis in HCV patients: a prospective study of 935 patients," Journal of Hepatology, vol. 46, no. 4, pp. 628-634, 2007.

[25] E. B. Tapper, E. B. Cohen, K. Patel et al., "Levels of alanine aminotransferase confound use of transient elastography to diagnose fibrosis in patients with chronic hepatitis $\mathrm{C}$ virus infection," Clinical Gastroenterology and Hepatology, vol. 10, no. 8, pp. 932.e1-937.e1, 2012.

[26] G. Millonig, F. M. Reimann, S. Friedrich et al., "Extrahepatic cholestasis increases liver stiffness (FibroScan) irrespective of fibrosis," Hepatology, vol. 48, no. 5, pp. 1718-1723, 2008.

[27] P. Lebray, S. Varnous, F. Charlotte, A. Varaut, T. Poynard, and V. Ratziu, "Liver stiffness is an unreliable marker of liver fibrosis in patients with cardiac insufficiency," Hepatology, vol. 48, no. 6, p. 2089, 2008.

[28] U. Arena, M. L. Platon, C. Stasi et al., "Liver stiffness is influenced by a standardized meal in patients with chronic hepatitis $\mathrm{C}$ virus at different stages of fibrotic evolution," Hepatology, vol. 58, no. 1, pp. 65-72, 2013.
[29] R. P. Myers, M. Elkashab, M. Ma, P. Crotty, and G. PomierLayrargues, "Transient elastography for the noninvasive assessment of liver fibrosis: a multicentre Canadian study," Canadian Journal of Gastroenterology, vol. 24, no. 11, pp. 661-670, 2010.

[30] J. Boursier, V. de Ledinghen, J.-P. Zarski et al., "Comparison of eight diagnostic algorithms for liver fibrosis in hepatitis C: new algorithms are more precise and entirely noninvasive," Hepatology, vol. 55, no. 1, pp. 58-67, 2012.

[31] N. H. Afdhal, S. Zeuzem, R. T. Schooley et al., “The new paradigm of hepatitis $\mathrm{C}$ therapy: integration of oral therapies into best practices," Journal of Viral Hepatitis, vol. 20, no. 11, pp. 745-760, 2013.

[32] Y. Shiratori, F. Imazeki, M. Moriyama et al., "Histologic improvement of fibrosis in patients with hepatitis $\mathrm{C}$ who have sustained response to interferon therapy," Annals of Internal Medicine, vol. 132, no. 7, pp. 517-524, 2000.

[33] A. J. van der Meer, B. J. Veldt, J. J. Feld et al., "Association between sustained virological response and all-cause mortality among patients with chronic hepatitis $\mathrm{C}$ and advanced hepatic fibrosis," The Journal of the American Medical Association, vol. 308, no. 24, pp. 2584-2593, 2012.

[34] E. Ogawa, N. Furusyo, K. Toyoda, H. Takeoka, S. Maeda, and J. Hayashi, "The longitudinal quantitative assessment by transient elastography of chronic hepatitis $\mathrm{C}$ patients treated with pegylated interferon alpha- $2 \mathrm{~b}$ and ribavirin," Antiviral Research, vol. 83, no. 2, pp. 127-134, 2009.

[35] C. Hézode, L. Castéra, F. Roudot-Thoraval et al., "Liver stiffness diminishes with antiviral response in chronic hepatitis C," Alimentary Pharmacology and Therapeutics, vol. 34, no. 6, pp. 656-663, 2011.

[36] R. D’Ambrosio, A. Aghemo, M. Fraquelli et al., "The diagnostic accuracy of Fibroscan for cirrhosis is influenced by liver morphometry in HCV patients with a sustained virological response," Journal of Hepatology, vol. 59, no. 2, pp. 251-256, 2013.

[37] G. L.-H. Wong, H. L.-Y. Chan, P. C.-L. Choi et al., "Noninvasive algorithm of enhanced liver fibrosis and liver stiffness measurement with transient elastography for advanced liver fibrosis in chronic hepatitis B," Alimentary Pharmacology and Therapeutics, vol. 39, no. 2, pp. 197-208, 2014.

[38] G. L.-H. Wong, V. W.-S. Wong, P. C.-L. Choi et al., "Increased liver stiffness measurement by transient elastography in severe acute exacerbation of chronic hepatitis B," Journal of Gastroenterology and Hepatology, vol. 24, no. 6, pp. 1002-1007, 2009.

[39] L. Castéra, P.-H. Bernard, B. Le Bail et al., "Transient elastography and biomarkers for liver fibrosis assessment and follow-up of inactive hepatitis B carriers," Alimentary Pharmacology and Therapeutics, vol. 33, no. 4, pp. 455-465, 2011.

[40] S. Maimone, V. Calvaruso, M. Pleguezuelo et al., "An evaluation of transient elastography in the discrimination of HBeAgnegative disease from inactive hepatitis B carriers," Journal of Viral Hepatitis, vol. 16, no. 11, pp. 769-774, 2009.

[41] H. L.-Y. Chan, G. L.-H. Wong, P. C.-L. Choi et al., "Alanine aminotransferase-based algorithms of liver stiffness measurement by transient elastography (Fibroscan) for liver fibrosis in chronic hepatitis B," Journal of Viral Hepatitis, vol. 16, no. 1, pp. 36-44, 2009.

[42] G. V. Papatheodoridis, S. Manolakopoulos, Y.-F. Liaw, and A. Lok, "Follow-up and indications for liver biopsy in HBeAgnegative chronic hepatitis B virus infection with persistently normal ALT: a systematic review," Journal of Hepatology, vol. 57, no. 1, pp. 196-202, 2012. 
[43] G. L.-H. Wong, V. W.-S. Wong, P. C.-L. Choi et al., "Evaluation of alanine transaminase and hepatitis B Virus DNA to predict liver cirrhosis in hepatitis $\mathrm{B}$ e antigen-negative chronic hepatitis B using transient elastography," American Journal of Gastroenterology, vol. 103, no. 12, pp. 3071-3081, 2008.

[44] G. L.-H. Wong, V. W.-S. Wong, P. C.-L. Choi et al., "Clinical factors associated with liver stiffness in hepatitis $\mathrm{B}$ e antigenpositive chronic hepatitis B patients," Clinical Gastroenterology and Hepatology, vol. 7, no. 2, pp. 227-233, 2009.

[45] P. Marcellin, E. Gane, M. Buti et al., "Regression of cirrhosis during treatment with tenofovir disoproxil fumarate for chronic hepatitis B: a 5-year open-label follow-up study," The Lancet, vol. 381, no. 9865, pp. 468-475, 2013.

[46] E. Ogawa, N. Furusyo, M. Murata et al., "Longitudinal assessment of liver stiffness by transient elastography for chronic hepatitis B patients treated with nucleoside analog," Hepatology Research, vol. 41, no. 12, pp. 1178-1188, 2011.

[47] Y.-H. Kuo, S.-N. Lu, C.-H. Chen et al., "The changes of liver stiffness and its associated factors for chronic hepatitis B patients with entecavir therapy," PLoS ONE, vol. 9, no. 3, Article ID e93160, 2014.

[48] G. L.-H. Wong, V. W.-S. Wong, P. C.-L. Choi et al., "Ontreatment monitoring of liver fibrosis with transient elastography in chronic hepatitis B patients," Antiviral Therapy, vol. 16, no. 2, pp. 165-172, 2011.

[49] M. N. Kim, S. U. Kim, B. K. Kim et al., "Increased risk of hepatocellular carcinoma in chronic hepatitis B patients with transient elastography-defined subclinical cirrhosis," Hepatology, vol. 61, no. 6, pp. 1851-1859, 2015.

[50] M. Fraquelli, C. Rigamonti, G. Casazza et al., "Reproducibility of transient elastography in the evaluation of liver fibrosis in patients with chronic liver disease," Gut, vol. 56, no. 7, pp. 968973, 2007.

[51] G. Ferraioli, C. Tinelli, B. Dal Bello, M. Zicchetti, G. Filice, and C. Filice, "Accuracy of real-time shear wave elastography for assessing liver fibrosis in chronic hepatitis C: a pilot study," Hepatology, vol. 56, no. 6, pp. 2125-2133, 2012.

[52] T. Poynard, M. Munteanu, E. Luckina et al., "Liver fibrosis evaluation using real-time shear wave elastography: applicability and diagnostic performance using methods without a gold standard," Journal of Hepatology, vol. 58, no. 5, pp. 928-935, 2013.

[53] C. Cassinotto, B. Lapuyade, A. Mouries et al., "Non-invasive assessment of liver fibrosis with impulse elastography: comparison of Supersonic Shear Imaging with ARFI and FibroScan," Journal of Hepatology, vol. 61, no. 3, pp. 550-557, 2014.

[54] M. Friedrich-Rust, J. Nierhoff, M. Lupsor et al., "Performance of Acoustic Radiation Force Impulse imaging for the staging of liver fibrosis: a pooled meta-analysis," Journal of Viral Hepatitis, vol. 19, no. 2, pp. e212-e219, 2012.

[55] I. Sporea, S. Bota, M. Peck-Radosavljevic et al., "Acoustic radiation force impulse elastography for fibrosis evaluation in patients with chronic hepatitis C: an international multicenter study," European Journal of Radiology, vol. 81, no. 12, pp. 41124118, 2012.

[56] S. Bota, H. Herkner, I. Sporea et al., "Meta-analysis: ARFI elastography versus transient elastography for the evaluation of liver fibrosis," Liver International, vol. 33, no. 8, pp. 1138-1147, 2013.

[57] J. Nierhoff, A. A. Chávez Ortiz, E. Herrmann, S. Zeuzem, and M. Friedrich-Rust, "The efficiency of acoustic radiation force impulse imaging for the staging of liver fibrosis: a metaanalysis," European Radiology, vol. 23, no. 11, pp. 3040-3053, 2013.

[58] É. Bavu, J.-L. Gennisson, M. Couade et al., "Noninvasive in vivo liver fibrosis evaluation using supersonic shear imaging: a clinical study on 113 hepatitis C virus patients," Ultrasound in Medicine and Biology, vol. 37, no. 9, pp. 1361-1373, 2011.

[59] K. Nightingale, S. McAleavey, and G. Trahey, "Shear-wave generation using acoustic radiation force: in vivo and ex vivo results," Ultrasound in Medicine and Biology, vol. 29, no. 12, pp. 1715-1723, 2003.

[60] M. D. Beland, S. F. Brown, J. T. Machan, R. J. Taliano, K. Promrat, and J. J. Cronan, "A pilot study estimating liver fibrosis with ultrasound shear-wave elastography: does the cause of liver disease or location of measurement affect performance?" American Journal of Roentgenology, vol. 203, no. 3, pp. W267W273, 2014.

[61] J. Bamber, D. Cosgrove, C. F. Dietrich et al., "EFSUMB guidelines and recommendations on the clinical use of ultrasound elastography. Part 1: basic principles and technology," Ultraschall in der Medizin, vol. 34, no. 2, pp. 169-184, 2013.

[62] A. Manduca, T. E. Oliphant, M. A. Dresner et al., "Magnetic resonance elastography: non-invasive mapping of tissue elasticity," Medical Image Analysis, vol. 5, no. 4, pp. 237-254, 2001.

[63] E. C. Ehman, P. J. Rossman, S. A. Kruse, A. V. Sahakian, and K. J. Glaser, "Vibration safety limits for magnetic resonance elastography," Physics in Medicine and Biology, vol. 53, no. 4, pp. 925-935, 2008.

[64] S. Singh, S. K. Venkatesh, Z. Wang et al., "Diagnostic performance of magnetic resonance elastography in staging liver fibrosis: a systematic review and meta-analysis of individual participant data," Clinical Gastroenterology and Hepatology, vol. 13, no. 3, pp. 440-451.e6, 2015.

[65] Y. Guo, S. Parthasarathy, P. Goyal, R. J. McCarthy, A. C. Larson, and F. H. Miller, "Magnetic resonance elastography and acoustic radiation force impulse for staging hepatic fibrosis: a metaanalysis," Abdominal Imaging, vol. 40, no. 4, pp. 818-834, 2015. 


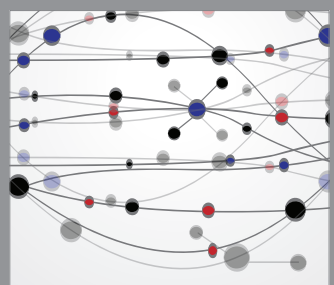

The Scientific World Journal
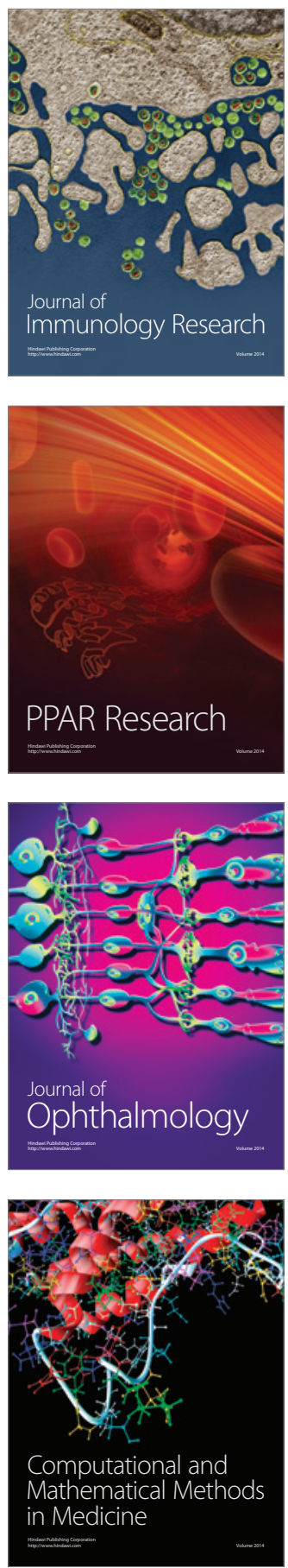

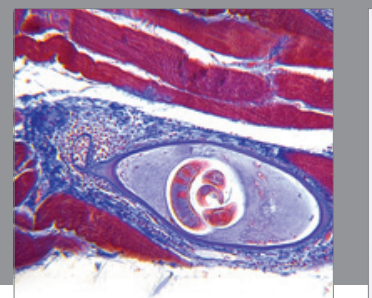

Gastroenterology

Research and Practice
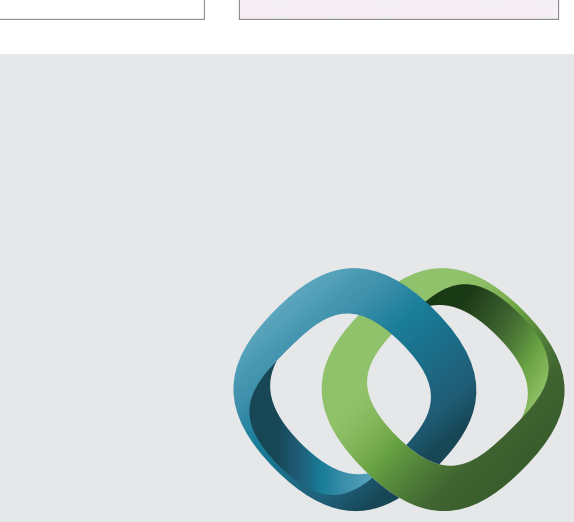

\section{Hindawi}

Submit your manuscripts at

http://www.hindawi.com
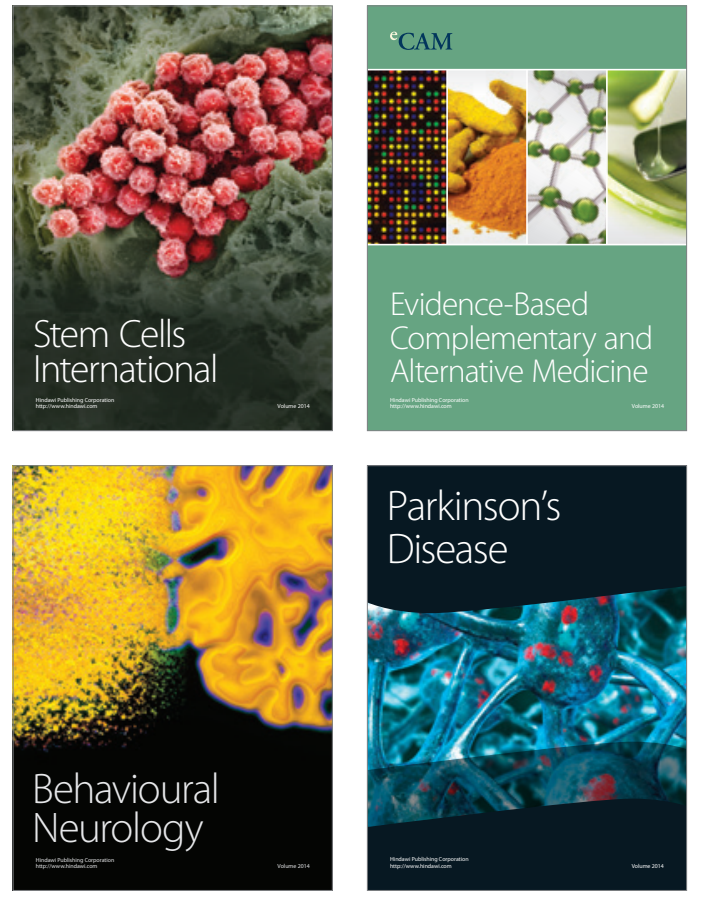
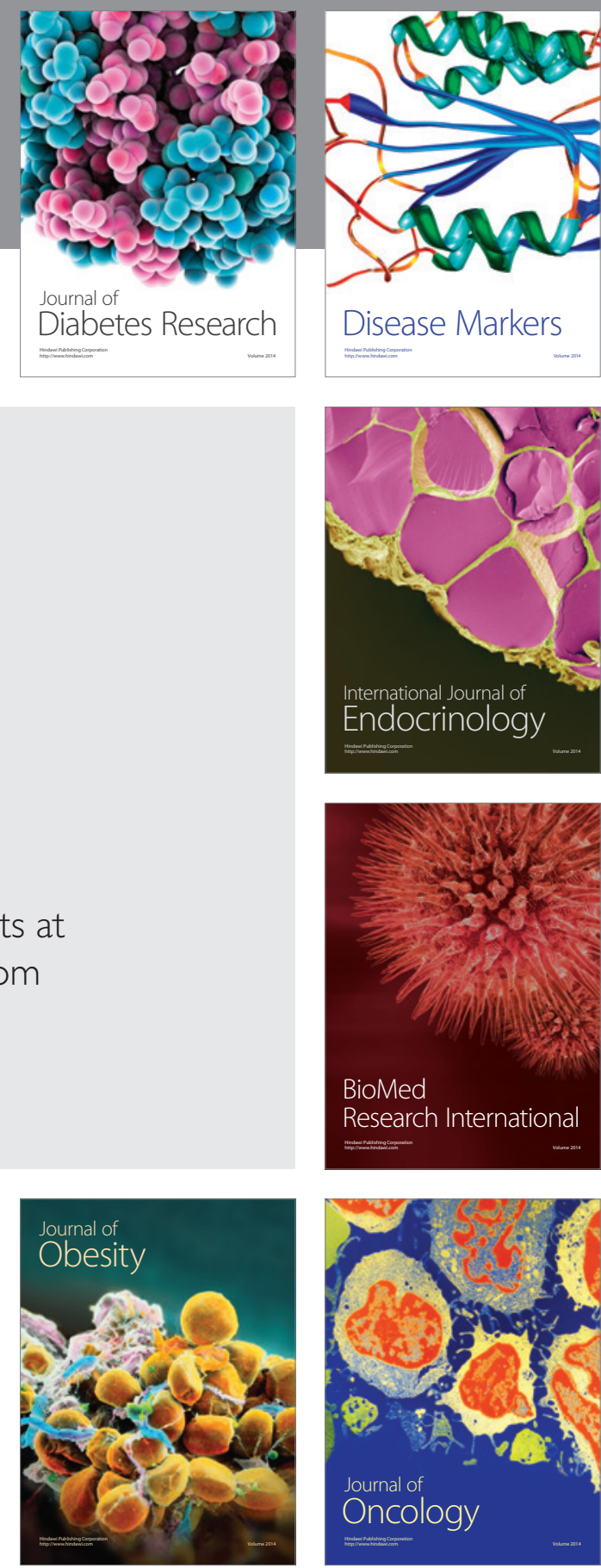

Disease Markers
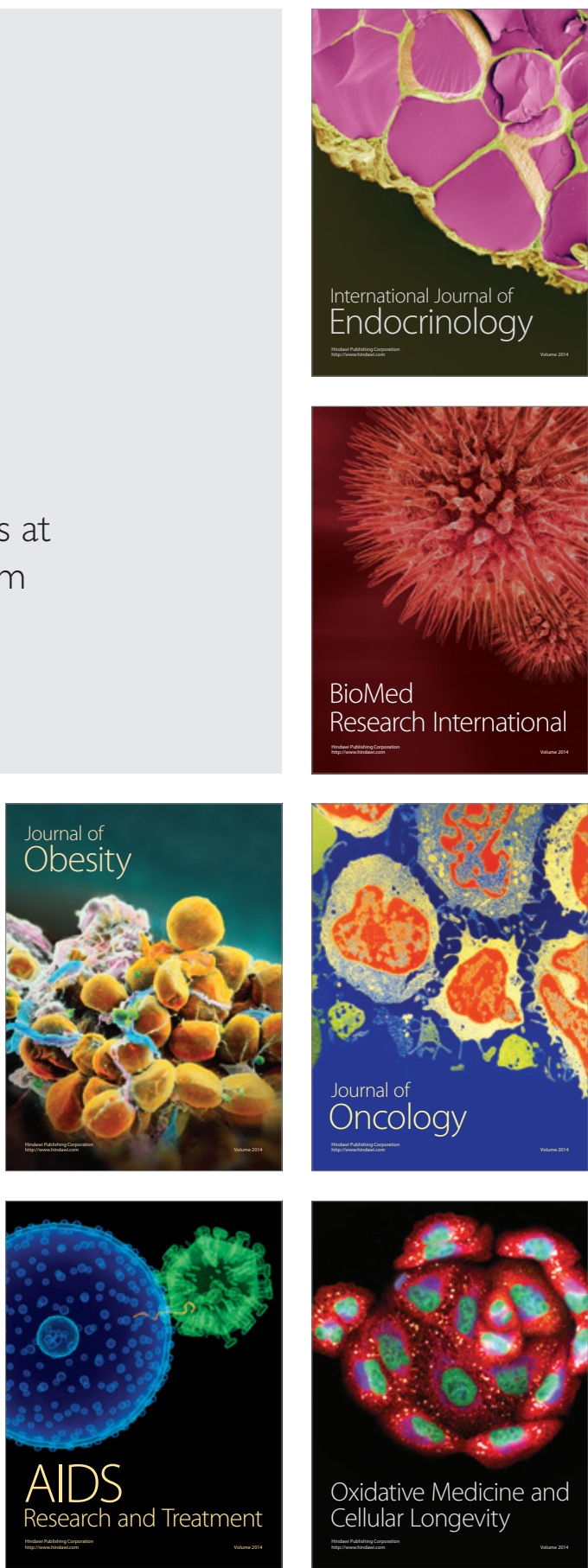